\title{
Unveiling the Orbital Angular Momentum and Acceleration of Electron Beams
}

\author{
Roy Shiloh, ${ }^{1, *}$ Yuval Tsur, ${ }^{1}$ Roei Remez, ${ }^{1}$ Yossi Lereah, ${ }^{1}$ Boris A. Malomed, ${ }^{1}$ Vladlen Shvedov, ${ }^{2}$ Cyril Hnatovsky, ${ }^{2}$ \\ Wieslaw Krolikowski, ${ }^{2}$ and Ady Arie ${ }^{1}$ \\ ${ }^{1}$ Department of Physical Electronics, Fleischman Faculty of Engineering, Tel Aviv University, Tel Aviv 6997801, Israel \\ ${ }^{2}$ Laser Physics Centre, The Australian National University, Canberra ACT 0200, Australia
}

(Received 27 November 2013; published 4 March 2015)

\begin{abstract}
New forms of electron beams have been intensively investigated recently, including vortex beams carrying orbital angular momentum, as well as Airy beams propagating along a parabolic trajectory. Their traits may be harnessed for applications in materials science, electron microscopy, and interferometry, and so it is important to measure their properties with ease. Here, we show how one may immediately quantify these beams' parameters without need for additional fabrication or nonstandard microscopic tools. Our experimental results are backed by numerical simulations and analytic derivation.
\end{abstract}

DOI: 10.1103/PhysRevLett.114.096102

PACS numbers: 68.37.Lp, 41.85.-p, 42.40.Jv

Introduction.-In the last few years, it became possible to generate special shapes of electron beams. One of these is the vortex beam [1-4] having a helical wave front structure and a phase singularity on axis. Its azimuthal phase dependence is $\exp (i l \phi)$, where integer $l$ is the topological charge and $\phi$ is the azimuthal angle. This beam carries an orbital angular momentum (OAM) of $\hbar l$ [5]. Another interesting beam is the Airy beam, having a transverse amplitude dependence in the form of the Airy function, $\operatorname{Ai}\left(x / x_{0}\right)$, where $x_{0}$ defines the transverse scale. It is shape preserving and moves along a parabolic trajectory in free space with a nodal trajectory coefficient, sometimes referred to as the "acceleration" coefficient, $\tau=1 /\left(2 k_{\mathrm{DB}}^{2} x_{0}^{3}\right)$, where $k_{\mathrm{DB}}$ is the (de Broglie) wave number. These beams, generated and observed in a transmission electron microscope (TEM) are expected to open new possibilities for interactions between electrons and matter, as well as for electron microscopy and interferometry. For example, vortex beams were used in electron energy loss spectroscopy in order to characterize the magnetic state of a ferromagnetic material [2], while Airy beams were proposed for realization of a new type of electron interferometer [6]. In order to utilize these new types of electron beams, it is required to develop methods that allow one to easily determine their defining properties - the OAM of a vortex beam or the nodal trajectory coefficient of an Airy beam. Here, we first demonstrate a straightforward method for single-mode OAM determination. The method relies on the astigmatic Fourier transform of the beam; the concept and measuring procedure we describe are central to our main discussion: the measurement of the nodal trajectory of electron Airy beams.

OAM determination.-In their 1991 article [7], Abramochkin and Volostnikov discussed the mathematics of beam transformations under astigmatic conditions. Since then, different authors proposed and demonstrated mode conversion in lasers [8], linear and nonlinear optics $[9,10]$, and free-electron beams in TEM $[11,12]$. In the latter works, McMorran et al. and Schattschneider et al. have shown in theory and experiment how Laguerre-Gauss modes transform to Hermite-Gauss modes. Recently, it was proposed, in light optics, that a cylindrical lens acting as a mode converter [13] be used to quantify the OAM of optical vortices [14]. Thus, a vortex of integer topological charge converts into a corresponding Hermite-Gauss-like mode, where the number of dark stripes precisely indicates the topological charge. The advantage of this method is in its simplicity and generality: the only addition to the setup is a cylindrical lens. In the TEM, the lens is inherent-the condenser and objective stigmators may be used to impose a strong astigmatism along a desired axis, thereby implementing the astigmatic transformation required to perform mode conversion.

To observe this phenomena, in our first experiment we designed a two-dimensional binary hologram-a fork grating which is mathematically written

$$
h(x, y)=\operatorname{sgn}\left\{\sin \left[\frac{2 \pi x}{\Lambda}-l \arctan 2(y, x)+\delta \frac{\pi}{2}\right]+\Delta\right\},
$$

where $2 \pi x / \Lambda$ is a linear grating along $x$, with period $\Lambda$, upon which the four-quadrant inverse-tangent function [15] modulates the spiral phase of the vortex, $l$ being the topological charge. $\delta$ reduces fabrication errors in the center by preserving continuity: its value is chosen equal to zero (one) if $l$ is odd (even). $\Delta=0.25$, related to duty cycle, is chosen so that both even and odd diffraction orders are visible. A Raith IonLine focused ion beam was used to mill into a $100 \mathrm{~nm}$ SiN membrane coated by $50 \mathrm{~nm} \mathrm{Au}$, from the gold side. This layer prevents charging of the sample and assures that the membrane behaves as an amplitude (binary) grating. 
A fork grating with $l=3$ topological charge was fabricated [Fig. 1(e)], based on a $\Lambda=0.750 \mu \mathrm{m}$ period, along with a linear (Bragg) grating of period $\Lambda=0.445 \mu \mathrm{m}$ which was fabricated for calibration purposes [Fig. 1(f)]. The sample was mounted onto a Tecnai F-20 FEG-TEM with a single tilt specimen holder and, subsequently, observed in the microscope in low angle diffraction mode through a $10 \mu \mathrm{m}$ aperture.

In order to form the far field diffraction pattern, the condenser lens was set to measure a focused spot. The fork grating was then aligned under the beam and the hologram imposed a spiral phase such that in each diffraction order $m$, a vortex of OAM $l m$ emerged [Fig. 1(a)]. The intense on-axis (zero-order) beam was blocked to prevent damage to the CCD.

Since the electron beam impinging on the hologram does not have a Gaussian distribution, the emerging vortices are not pure Laguerre-Gauss modes. However, the resulting diffraction pattern is undeniably dominated by solutions of the Schrödinger equation

$$
\left[\nabla_{\perp}^{2}+2 i k_{\mathrm{DB}} \frac{\partial}{\partial z}\right] \psi=0
$$

where $k_{\mathrm{DB}}=1 / \lambda_{\mathrm{DB}}$ is the de Broglie wave number. This equation governs the paraxial dynamics of the slowly varying envelope of the free electron beam in the TEM [16], a statement verified by the results of our next step.

The electron beam is made sufficiently astigmatic along the desired axis so it becomes elliptic; in the present case, this axis must be parallel to the lateral direction of the fork. As a reference, the linear grating was first measured under these conditions. As will be explained below, we purposely measured slightly out of the focal plane [Fig. 1(c)]. This reference serves to assess the efficacy of the astigmatic transformation. When the beam is sufficiently astigmatic, the vortices in the different diffraction orders are converted into Hermite-Gauss-like modes [Fig. 1(b)], and the resulting dark stripes indicate the OAM carried by each order. In addition, the angle of the transformed orders signifies the rotation direction of the vortex: clockwise or counterclockwise. The linear grating's diffraction spots become lines, as expected from a cylindrical lens [Fig. 1(d)]. Note that, if the linear grating's diffraction pattern was observed in focus rather than out of it, the spots would be so small that the equipped Gatan 694 CCD would not be able to distinguish the ellipticity of the beam; also, the additional diffraction lines visible in the negative orders [Fig. 1(d)] are a result of asymmetry of the sample relative to the beam, introduced by using a single-tilt rather than a double-tilt holder. In the Supplemental Material [17], we show a similar, additional measurement of at least $10 \hbar$. A potential application of this method would be the

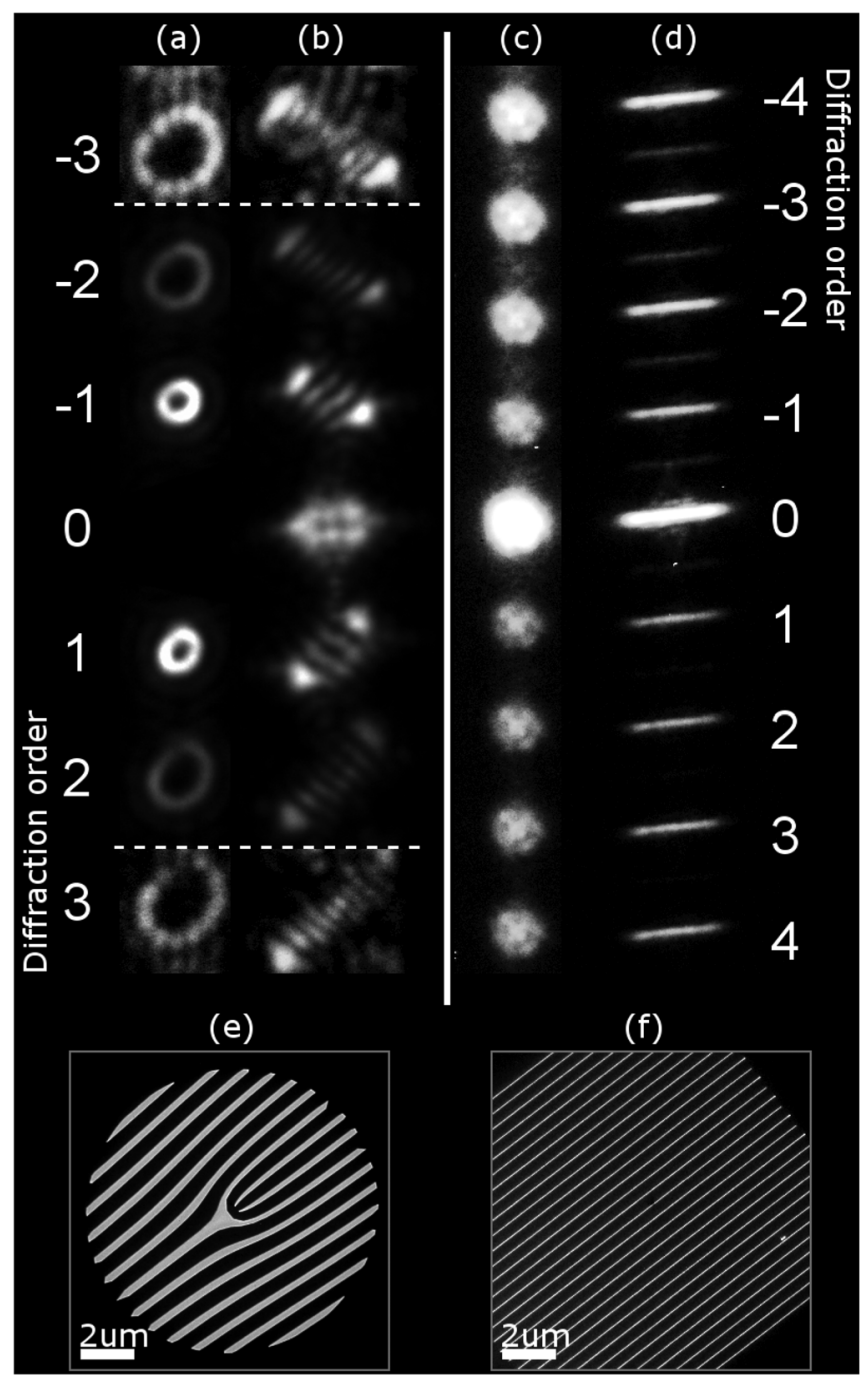

FIG. 1. Experimental results: diffraction patterns of (a) vortices generated by a charge 3 fork grating, using a focused stigmatic beam (zero-order blocked); (b) corresponding vortices after astigmatic transformation; (c) diffraction pattern generated by a linear calibration grating using a stigmatic beam, out of focus; (d) corresponding lines resulting from the astigmatic transformation, out of focus. Below: TEM images of (e) charge 3 fork $(0.750 \mu \mathrm{m}$ period); (f) linear grating $(0.445 \mu \mathrm{m}$ period $)$. Note: brightness levels in the third order parts, as marked by the dashed lines in (a) and (b), have been modified for visibility.

characterization of localized defects in materials: previously, it was shown that spontaneously stacked graphite films can be modeled as spiral phase plates for electrons and, therefore, add OAM to the electron beam [3], but, in order to characterize this OAM, an interference measurement was needed. The astigmatic transformation method presented here provides a simpler and more direct measurement of the direction of helicity and the thickness variation of the defect. Moreover, the electron energy can be tuned to achieve a $2 \pi$ phase step for the spiral phase plate. 
Determining the Airy beam's nodal trajectory.-Optical generation and manipulation of Airy beams $[18,19]$ has been recently under the spot-light; here, we present, to the best of our knowledge, the first example of Airy beams with special transformations, specifically, the astigmatic transformation. It is of interest to measure the nodal trajectory coefficient of 2D Airy beams; in order to do so from a stigmatic pattern, one must either record the propagation of the Airy beam in different planes and measure the actual trajectory, or directly measure the density of the Airy lobes and relate it to the length scale $x_{0}$ (for more details, see [6]). These two methods are, unfortunately, time consuming and arduous; the latter case, for example, is generally dangerous to perform experimentally, since such a measurement requires the pattern to be in focus, to exhibit high contrast and low signal-to-noise ratio so as to make the lobes discernible and measurable with precision, all the while protecting the CCD camera from damage. Using astigmatic transformation, we show that the energy of the beam is distributed over a large area and the beam's acceleration coefficient is deduced from the curvature of the generated pattern.

For the purpose of generality, we express the transformation of a beam, $f(x, y)$, under general astigmatic conditions by [[7], Eq. (8)]

$$
F\left(\theta_{x}, \theta_{y}, a\right)=\int_{-\infty}^{\infty} \int_{-\infty}^{\infty} e^{i\left(\theta_{x} x+\theta_{y} y\right)+i \psi(x, y, a, \alpha)} f(x, y) d x d y .
$$

Here, we define the Cartesian coordinates $(x, y)$ in the plane of the holographic mask, and similarly, the geometric angles $\left(\theta_{x}, \theta_{y}\right)$ in the diffraction plane. $\psi(x, y, a, \alpha)=$ $a\left[\left(x^{2}-y^{2}\right) \cos 2 \alpha+2 x y \sin 2 \alpha\right], a$ is the measure of astigmatism and $\alpha$ is a rotation angle with respect to the curve $y=x$. In Ref. [7], it is shown that, in the special case of $\psi(x, y, a, \alpha=\pi / 4)=2 a x y$, Hermite-Gauss and LaguerreGauss beams interchange; this mathematical paradigm explains our previous results.

Applying the transformation to Airy beams, we write the Fourier transform

$F\left(\theta_{x}, \theta_{y}, a\right)=\int_{-\infty}^{\infty} \int_{-\infty}^{\infty} e^{i\left(\theta_{x} x+\theta_{y} y\right)+i 2 a x y} e^{i\left(3 x_{0}^{3}\right)^{-1}\left(x^{3}+y^{3}\right)} d x d y$,

which yields an Airy beam in the diffraction plane. Note that $f(x, y)=\exp \left[i\left(3 x_{0}^{3}\right)^{-1}\left(x^{3}+y^{3}\right)\right]$ is a cubic phase term and $x_{0}$ is the Airy-related transverse scale. Figure 2 depicts numerical simulations for constant astigmatism $a=4 \mu \mathrm{m}^{-2}$ and two different values of $x_{0}$, with the main (high-intensity) lobes marked; the curvature of these lobes is related to $a$ and $x_{0}$, and they, in turn, are related to the nodal trajectory; thus, a method of finding these parameters in situ could be devised. While

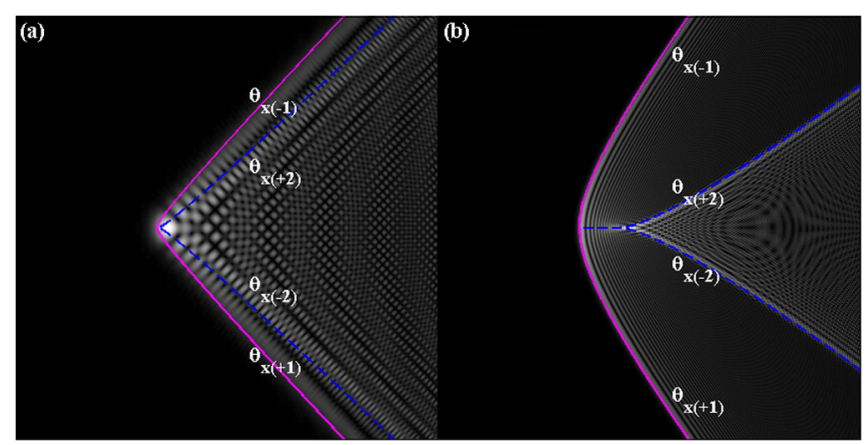

FIG. 2 (color online). Examples of astigmatic Airy beams with different $x_{0}$ values and astigmatic work point $a=4 \mu \mathrm{m}^{-2}$. In the left panel, low $x_{0}$ manifests in a shape very similar to the familiar Airy, while in the right panel the high acceleration (in conjunction with the value of $a$ ) yields an astigmatic Airy. The magenta and blue lines mark the $\theta_{x(\mp 1)}$ and $\theta_{x( \pm 2)}$ solutions, respectively. (a) $x_{0}=0.21 \mu \mathrm{m}$ and (b) $x_{0}=0.63 \mu \mathrm{m}$.

calculation of the Fourier integral in Eq. (4) in the stigmatic $(a=0)$ case is easy and results in the twodimensional Airy pattern, obtaining a closed-form analytic solution to the astigmatic $(a \neq 0)$ case is difficult. However, one can describe the formation of the diffraction pattern and particularly the main lobes using geometric optics.

In this approach, we perform ray-tracing analysis of the cubic phase imposed by the holographic mask and the phase of the impinging, astigmatic beam, and seek an analytic expression for the main lobes. To reduce the complexity of the derivation, we utilize the symmetry of the astigmatic Airy and the invariance of the pattern to additional focus and find that the four high-intensity lobes of the astigmatic Airy are described geometrically by

$$
\begin{array}{ll}
\left(a^{2} x_{0}^{3}\right)^{-1} \theta_{x(\mp 1)}=\mp(3+\cosh u)^{3 / 2} \sinh \frac{u}{2} & \text { Outer lobes, } \\
\left(a^{2} x_{0}^{3}\right)^{-1} \theta_{x( \pm 2)}= \pm(-3+\cosh u)^{3 / 2} \cosh \frac{u}{2} & \text { Inner lobes, }
\end{array}
$$

where $\sqrt{2} \theta_{y}=\left(a^{2} x_{0}^{3}\right) \sinh ^{2} u$, and $\theta_{x}, \theta_{y}$ are the coordinates in Fourier space. Here, each of the top and bottom rows represents the two outer and inner curves, respectively, as depicted in Fig. 2. It is worth noting that, since our derivation is based on geometric optics, there is no theoretical limitation to our results as long as the Airy beam is generated through an aperture large enough with respect to $x_{0}$, such that enough oscillations of the Airy envelope are transmitted. The full mathematical details are in the Supplemental Material [17].

To see this in experiment, we designed binary holographic masks of varying diameters $(20-80 \mu \mathrm{m})$ using the expression

$$
h(x, y)=\operatorname{sign}\left\{\cos \left[\left(x^{3}+y^{3}\right) / 3 x_{0}^{3}\right]\right\} .
$$


The holographic masks were fabricated on a membrane similar to that of the vortex masks, with $x_{0}$ ranging $\left(0.33 \leq \mathrm{x}_{0} \leq 1.6\right) \times 10^{-6} \mathrm{~m}$, some of which are depicted in Fig. 3; our considerations in selecting these values were purely technical and are based on our FIB's fabrication capability, but the method itself is in no way limited by these values. The cubic phase modulation imposed on the beam yields an Airy pattern in the diffraction plane. Using the TEM's stigmators, we set the astigmatism to a constant value and measure the curved lobes of the resulting astigmatic Airy patterns in proximity to the diffraction plane, as seen in Figs. 3(a)-3(d). Note that, contrary to the simulation, in experiment, we find a mirror-symmetric astigmatic Airy pattern around the astigmatic axis; the two patterns are the real and virtual image of our inline holographic mask, and appear so, in part, due to the binary mask fabrication.

The value of the astigmatism is related to $a$, which can be referred to as the "astigmatic working point", can be chosen appropriately to make the measurement of a range of $x_{0}$ easier: as we have shown earlier, upon normalization the astigmatic Airy is shape preserving and depends linearly on the scaling $a^{2} x_{0}^{3}$; thus, if $a$ is too small, one would need a very large value of $x_{0}$ to observe the astigmatic Airy, and if $a$ is too large, moderate values of $x_{0}$ might yield a pattern whose details are impossible to discern. Different methods of determining $a$ could be devised; here, we will deduce it from our series of measurements: as analyzed in Figs. 3(e)-3(f), through linear fitting, we deduce $a=3.46 \pm 0.09 \mu \mathrm{m}^{-2}$. This value, calibrated by our measurement of known $x_{0}$ values, may now be used for the measurement of unknown $x_{0}$ for other Airy beams and, subsequently, the nodal trajectory $\tau=1 /\left(2 k_{\mathrm{DB}}^{2} x_{0}^{3}\right)$, which in our case ranges $(7.7 \leq \tau \leq 876) \times 10^{-7} \mathrm{~m}^{-1}$.

The astigmatic work point gives us a range of measurable $x_{0}$ which spans at least 1 order of magnitude (in our experiment), a range that may be extended with an additional working point or simply a camera with higher resolution. One may, otherwise, devise a relation between the stigmator settings and $a$, and be free to measure Airy beams with nearly arbitrary $x_{0}$.

In this Letter, we first examined a method of measuring the OAM of electron vortex beams by means of astigmatic transformation, previously employed in light optics using cylindrical lenses. The transformation is applied, literally, by the turn of a knob in any standard TEM by correctly manipulating the lens stigmators. We extended the scope of this transformation and applied it to Airy beams in simulation and experiment, and found an analytic, closed-form expression [Eq. (5)] without any approximations, out of which the curvature of the resulting astigmatic shape and the Airy beam's nodal trajectory may be found. In effect, following calibration, this is a viable method of finding the nodal trajectory, or acceleration coefficient, $\tau$,

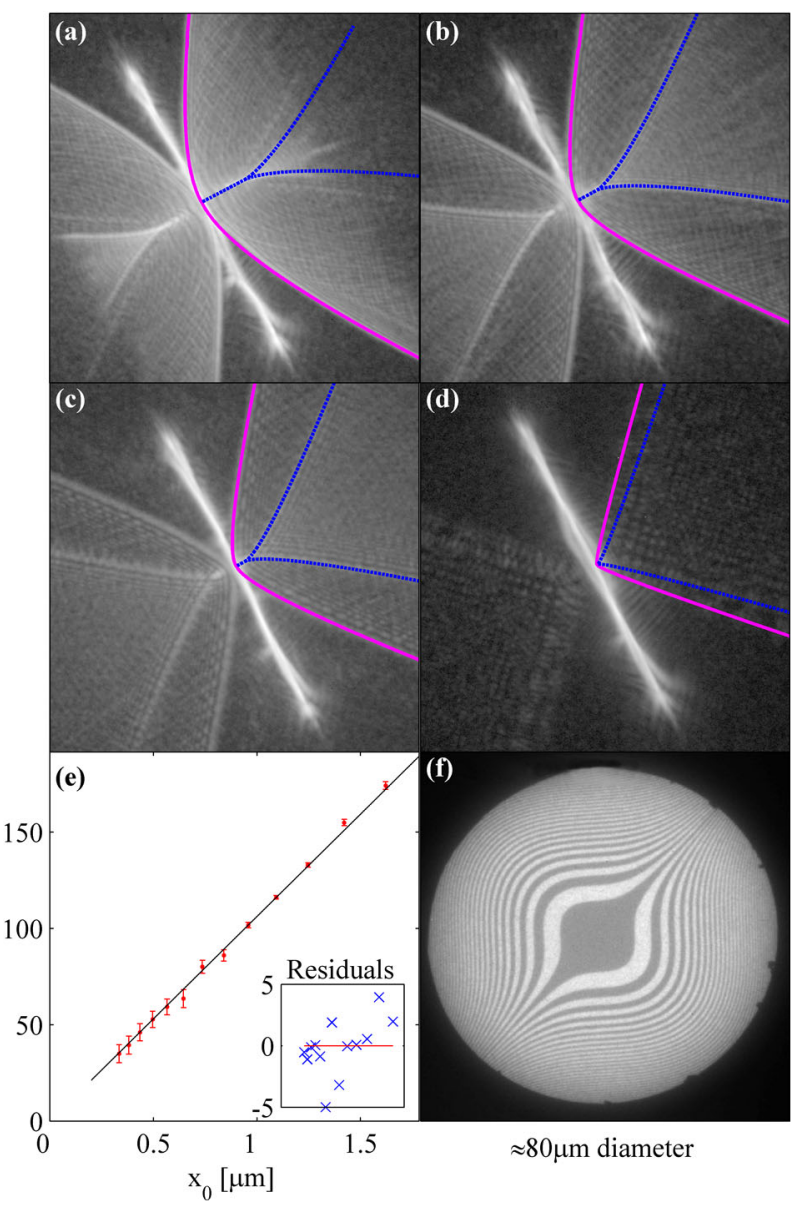

FIG. 3 (color online). Measurements of several astigmatic Airy beams with different $x_{0}$ values and corresponding fitted curves of the high-intensity main lobes. The only fitting parameter here is $a^{2 / 3} x_{0}$. From these measurements, we deduce the value of $a$ with our theoretical considerations. In (a), we may scarcely observe an additional, much weaker diffraction order, which is a result of the binary nature of the grating. Our linear fit in (e), yielding $a=3.46 \pm 0.09 \mu \mathrm{m}^{-2}$, is reinforced by the distribution observed in the residuals (inset) and figure of merit $R^{2}=0.9965$. The errors were derived from a $95 \%$ confidence interval scheme. (f) Example of a fabricated Airy mask. The field of view in these images is $100 \mu \mathrm{m}^{-1}$; the brightness and contrast were altered for visibility. (a) $x_{0}=1.2 \mu \mathrm{m}$, (b) $x_{0}=0.96 \mu \mathrm{m}$, (c) $x_{0}=0.74 \mu \mathrm{m}$, (d) $x_{0}=0.33 \mu \mathrm{m}$, (e) Fit to $a^{2 / 3} x_{0}\left[\mathrm{~m}^{-1 / 3}\right]$.

from one simple measurement. Our results warrant further theoretical investigation into the astigmatic transform of other beams to which it may be relevant, such as Bessel beams [20], parabolic beams [21], and beams with arbitrary caustic curves [22-24], unveiling their underlying propagation parameters.

The authors would like to acknowledge Dr. Yigal Lilach for his support in the fabrication process. The work was supported by the Israel Science Foundation, Grant No. 1310/13, by the German-Israeli Project Cooperation (DIP), and by the Australian Research Council. 
*royshilo@post.tau.ac.il

[1] K. Y. Bliokh, Y. P. Bliokh, S. Savel'ev, and F. Nori, Phys. Rev. Lett. 99, 190404 (2007).

[2] J. Verbeeck, H. Tian, and P. Schattschneider, Nature (London) 467, 301 (2010).

[3] M. Uchida and A. Tonomura, Nature (London) 464, 737 (2010).

[4] B. J. McMorran, A. Agrawal, I. M. Anderson, A. A. Herzing, H. J. Lezec, J. J. McClelland, and J. Unguris, Science 331, 192 (2011).

[5] L. Allen, M. W. Beijersbergen, R. J. C. Spreeuw, and J. P. Woerdman, Phys. Rev. A 45, 8185 (1992).

[6] N. Voloch-Bloch, Y. Lereah, Y. Lilach, A. Gover, and A. Arie, Nature (London) 494, 331 (2013).

[7] E. Abramochkin and V. Volostnikov, Opt. Commun. 83, 123 (1991).

[8] G. Machavariani, A. A. Ishaaya, L. Shimshi, N. Davidson, A. A. Friesem, and E. Hasman, Appl. Opt. 43, 2561 (2004).

[9] T. Ellenbogen, I. Dolev, and A. Arie, Opt. Lett. 33, 1207 (2008).

[10] N. V. Bloch, K. Shemer, A. Shapira, R. Shiloh, I. Juwiler, and A. Arie, Phys. Rev. Lett. 108, 233902 (2012).

[11] B. McMorran, A. Agrawal, I. M. Anderson, A. A. Herzing, H. Lezec, J. J. McClelland, and J. Unguris, in CLEO:2011 Laser Applications to Photonic Applications (IEEE, Piscataway, NJ, 2011), p. QMA1.

[12] P. Schattschneider, M. Stöger-Pollach, and J. Verbeeck, Phys. Rev. Lett. 109, 084801 (2012).
[13] V. G. Shvedov, C. Hnatovsky, W. Krolikowski, and A. V. Rode, Opt. Lett. 35, 2660 (2010).

[14] V. Denisenko, V. Shvedov, A. S. Desyatnikov, D. N. Neshev, W. Krolikowski, A. Volyar, M. Soskin, and Y. S. Kivshar, Opt. Express 17, 23374 (2009).

[15] $\arctan 2(y, x)$ returns the angle in the interval $[-\pi, \pi]$ as opposed to arctan which is limited to $[-\pi / 2, \pi / 2]$, i.e., only the two quadrants in the positive $x$ half-space.

[16] L. Reimer and H. Kohl, Transmission Electron Microscopy - Physics of Image Formation 5th ed. (Springer, New York, 2008).

[17] See Supplemental Material at http://link.aps.org/ supplemental/10.1103/PhysRevLett.114.096102 for mathematical derivations and additional measurements.

[18] M. V. Berry, Am. J. Phys. 47, 264 (1979).

[19] G. A. Siviloglou and D. N. Christodoulides, Opt. Lett. 32, 979 (2007).

[20] V. Grillo, E. Karimi, G. C. Gazzadi, S. Frabboni, M. R. Dennis, and R.W. Boyd, Phys. Rev. X 4, 011013 (2014).

[21] M. A. Bandres, Opt. Lett. 33, 1678 (2008).

[22] E. Greenfield, M. Segev, W. Walasik, and O. Raz, Phys. Rev. Lett. 106, 213902 (2011).

[23] L. Froehly, F. Courvoisier, A. Mathis, M. Jacquot, L. Furfaro, R. Giust, P. A. Lacourt, and J. M. Dudley, Opt. Express 19, 16455 (2011).

[24] I. Epstein and A. Arie, Phys. Rev. Lett. 112, 023903 (2014). 